\title{
Erfolge durch Kooperation im Bereich der Intervention bei häuslicher Gewalt in Deutschland - Ergebnisse neuerer Forschung
}

\author{
Ass. jur. Heike Rabe, Prof. Dr. Barbara Kavemann
}

$\int$ ie Präsenz von Gewalt in Ehe und Beziehungen (häusliche Gewalt) hat europaweit in den letzten Jahren einen höheren Platz auf der politischen Agenda eingenommen. Bisher beispiellos geblieben ist die Zusammenstellung eines Gesamtpaketes von Rahmenbedingungen für das rechtliche und psychosoziale Zusammenspiel bei der Bekämpfung von häuslicher Gewalt in Österreich (Dearing/Haller 2000). Unter der Bezeichnung Gewaltschutzgesetz ${ }^{1}$ wurden dort die zivilrechtliche Möglichkeit der Wohnungszuweisung, die polizeirechtliche Befugnis der Wegweisung/Betretungsverbot verknüpft und Interventionsstellen etabliert. Dieses Paket bietet den Betroffenen einen Anspruch auf lückenlosen Schutz vom ersten polizeilichen Einsatz bis hin zur Überlassung einer gemeinsamen Wohnung zur alleinigen Nutzung durch das Opfer.

Aufgrund der Verteilung von Gesetzgebungskompetenzen auf den Bund und die Länder musste man in Deutschland zur Umsetzung dieser Idee andere Wege gehen. Auf Bundesebene wurde auch hier ein Gewaltschutzgesetz ${ }^{2}$ verabschiedet, die Länder haben mittlerweile fast alle polizeiliche Spezialbefugnisse in die Polizeigesetze integriert. ${ }^{3}$ Psychosoziale Beratungsangebote für Geschädigte häuslicher Gewalt werden in unterschiedlicher Trägerschaft überwiegend pro aktiv in den Ländern organisiert.

Die Entwicklung in Deutschland ist sukzessiver verlaufen, die Umsetzung der Idee einer lückenlosen Interventionskette heterogener gestaltet als in Österreich, der Entwicklungsstand der Intervention bei häuslicher Gewalt dürfte sich aber mittlerweile in beiden Ländern angeglichen haben. Nicht nur der Aufbau von interinstitutioneller und interdisziplinärer Kooperation, sondern auch deren Evaluation ist - regional unterschiedlich intensiv - bereits erfolgt. Aufgrund von Untersuchungen ist mittlerweile eine differenzierte Schwachstellenanalyse möglich.

\section{1. Überblick über die Landschaft der Interventionsprojekte und Inter- ventionsstellen}

In den Jahren 1999 bis 2004 hat die Forschungsgruppe WiBIG im Auftrag des BMFSFJ 4 insgesamt zehn Interventionsprojekte wissenschaftlich begleitet. 5

Als Interventionsprojekte werden in Deutschland institutionalisierte Kooperationsbündnisse bezeichnet, die interinstitutionell und interdisziplinär arbeiten. Sie bündeln im Optimalfall Vertreterinnen und Vertreter aller Einrichtungen, Institutionen, Projekte und Professionen einer Region, die explizit gegen häusliche Gewalt arbeiten oder dafür gesellschaftliche Verantwortung tragen.

Im Zentrum ihrer Aktivitäten steht in der Regel ein zentrales Kooperationsgremium, wie z.B. ein Runder Tisch. Dieser unterscheidet sich von bereits bekannten und vielerorts aktiven fachspezifisch arbeitenden Runden Tischen dadurch, dass die hieran Beteiligten nicht in erster Linie als individuelle, interessierte Fachleute, sondern als Delegierte ihrer Institution mit einem Auftrag ihrer Institution teilnehmen. Es geht langfristig darum, dass nicht nur Einzelne unter Einbeziehung von interdisziplinärem Wissen ihre Praxis verbessern, sondern dass ganze Institutionen ein gleiches Verständnis von häuslicher Gewalt und gleiche Ziele entwickeln und auf dieser Grundlage ihre Verfahrensweisen aufeinander abstimmen.

In Deutschland entwickeln sich zunehmend solche Interventionsprojekte. Sie haben den Weg bereitet für eine veränderte Praxis bei häuslicher Gewalt. Sie haben Strukturen und Arbeitsroutinen innerhalb von bestehenden Einrichtungen und Institutionen verändert aber auch die Errichtung neuer Einrichtungen im Bereich von Beratung für Frauen und Männer initiiert.

Mittlerweile gibt es flächendeckend spezifische Beratungsstellen für Geschädigte häus- licher Gewalt nach polizeilicher Intervention in zwei Bundesländern (Mecklenburg-Vorpommern und Rheinlandpfalz). Andere Länder wie z. B. Niedersachen, Schleswig-Holstein und Sachsen-Anhalt bereiten gerade die landesweite Versorgung vor oder arbeiten wie Berlin an einem geeigneten Konzept. Im Folgenden einige Beispiele für diese dynamische Entwicklung. In Mecklenburg-Vorpommern ${ }^{6}$ wurden in jeder der fünf Polizeidirektionen im Land eine Interventionsstelle (IST) nach österreichischem Vorbild eingerichtet, die von der Frauen- und Gleichstellungsbeauftragten der Landesregierung Mecklenburg-Vorpommerns finanziert wird, wobei sich die Finanzierung aus Landesmitteln direkt aus dem Auftrag der Gefahrenabwehr ableitet. Hierfür sind die IST mit einem staatlichen Auftrag ausgestattet, der an die formale Anerkennung durch die Frauen- und Gleichstellungsbeauftragte der Landesregierung gekoppelt ist. ${ }^{7}$

Ein den IST in Mecklenburg-Vorpommern vergleichbares Modell, dessen Arbeit ebenfalls durch ländliche Strukturen geprägt ist, findet sich in Niedersachsen. Dort wurden im Rahmen des landesweiten »Aktionsplans zur Bekämpfung von Gewalt gegen Frauen« für die Beratung nach einer polizeilichen Wegweisung bei häuslicher Gewalt in sechs ländlichen Regionen so genannte Beratungs- und Interventionsstellen für Opfer häuslicher Gewalt (BISS) eingerichtet. Das Niedersächsische Ministerium für Frauen, Arbeit und Soziales finanzierte hiermit einen Modellversuch mit einer Laufzeit von drei Jahren (2002-2004) und dessen Evaluation (KFN 2005). Die positiven Ergebnisse hatten eine Ankündigung des Ministeriums zur Folge, die Finanzierung für eine flächendeckende Errichtung von BISS in ganz Niedersachsen zu übernehmen.

In einigen Bundesländern wurden neue Einrichtungen geschaffen, um die Beratungsaufgabe zu übernehmen. Anderenorts wurden bestehende Einrichtungen genutzt. Ein Modell war die Übertragung von Erstberatung bei häuslicher Gewalt nach polizeilicher Intervention auf den 
ASD, wie z. B. in Stuttgart oder Bremen (WiBIG 2004 a S.179 ff.). Hintergrund für diese Entscheidung waren vor allem datenschutzrechtliche Gründe, die eine Übermittlung personenbezogener Daten an freie Träger nicht zulassen.

\section{Forschungsstand in Deutschland}

Im Sommer 2004 wurden die Ergebnisse dreier neuer Studien, in Auftrag gegeben durch das Bundesministerium für Familie, Senioren, Frauen und Jugend (BMFSFJ), der Öffentlichkeit vorgestellt. ${ }^{8}$

Die Studie zur »Lebenssituation, Sicherheit und Gesundheit von Frauen in Deutschland « 9 eine repräsentative Befragung von 10.000 Frauen - legt erstmalig verlässliche, belastbare Zahlen zur Prävalenz und Inzidenz von Gewalt gegen Frauen vor. Die Bundesregierung setze damit einen Beschluss des Europarates um, dem andere europäische Länder bereits gefolgt waren. ${ }^{10}$ Die Studie wurde methodisch sorgfältig und in Abstimmung mit anderen Untersuchungen wie z.B. der Bristish Crime Survey durchgeführt und stieß auf großes nationales und internationales Interesse bei Forschenden und Praktiker/innen im Feld der Intervention und Prävention von Gewalt im privaten Raum. Die Angabe zur Prävalenz von Gewalt gegen Frauen in Ehe und Beziehung bedeutet selbstverständlich nicht, dass jede vierte Frau in einer Misshandlungsbeziehung lebt, sondern gibt Auskunft darüber, dass 25\% aller Frauen mindestens einmal im Leben Gewalt durch einen aktuellen oder ehemaligen Beziehungspartner erlebt haben. Diese Angabe korrespondiert mit den Ergebnissen anderer westeuropäischer Studien (Müller/Schröttle 2004).

Ebenfalls durch das BMFSFJ in Auftrag gegeben wurde die Pilotstudie "Gewalt gegen Männer «.11 Auch diese explorative Untersuchung von knapp 300 Männern weist einen Anteil von 25\% der Befragten aus, die mindestens einmal einen Akt der Aggression durch die aktuelle oder ehemalige Partnerin erlebt haben. Über geschlechtsspezifische Unterschiede in Kontext, Erleben und (Verletzungs-)Folgen der Gewalt gibt ein vergleichender Blick auf die Studien Aufschluss.

Die dritte im Sommer 2004 vorgelegte Untersuchung im Auftrag des BMFSFJ, die bereits oben erwähnte Studie »Gemeinsam gegen häusliche Gewalt - Kooperation, Intervention, Begleitforschung «, ist von anderer Natur: Über mehrere Jahre hinweg wurde von der Forschungsgruppe WiBIG die Entwicklung und Umgestaltung der Intervention und Unterstützung bei häuslicher Gewalt in Deutschland, der Aufbau interinstitutioneller Kooperation und die Einrichtung von Interventionsstellen wissenschaftlich begleitet. Am Beispiel von 10 Interventionsprojekten gegen häusliche Gewalt konnten neue Interventionsstrategien wie die polizeiliche Wegweisung und neuartige Unterstützungsangebote wie die pro-aktive Beratung und die Mobile Intervention evaluiert werden, eine Aktenauswertung bei den Amtsanwaltschaften an zwei Standorten analysierte Veränderungsbedarf. Mit dieser Forschung wurden der Praxis wertvolle Erkenntnisse zur Verfügung gestellt und eine erste Rückmeldung über die Wirksamkeit der neuen Strategien bei der Optimierung von Schutz und Intervention bei häuslicher Gewalt gegeben. Die Veränderungen in diesem Feld gehen vielerorts in großen Schritten voran, die Zahl der Interventionsprojekte und Interventionsstellen steigt, auch Angebote für gewalttätige Männer und mitbetroffene Kinder und Jugendliche entstehen zunehmend. ${ }^{12}$ Die WiBIG-Studie, die Elemente guter Praxis und gelingender Kooperation herausarbeitete, wurde ebenfalls mit großem nationalen und internationalen Interesse aufgenommen. ${ }^{13}$

Bereits jetzt zeigt sich sowohl der Nutzen dieser neuen Forschungen für die Weiterentwicklung der Praxis als auch weiterer Forschungsbedarf.

\section{Ergebnisse WiBIG}

Zur Untersuchung von veränderter Praxis führte WiBIG qualitative und quantitative Erhebungen in den verschiedenen Arbeitsbereichen einzelner Kooperationspartner der eingangs genannten Interventionsprojekte durch ${ }^{14}$. Die Ergebnisse zeigen, welche Veränderungen möglich sind, sie markieren aber auch Bereiche, in denen die Umsetzung von Veränderung besondere Schwierigkeiten bereitet. Sie lassen keine Aussagen über den Praxisstand in Einrichtungen, Institutionen und Behörden zu, die nicht im Kontext von Kooperationsbündnissen arbeiten.

\subsection{Erfolge von Interventionsprojekten}

Interventionsprojekte waren die ersten Kooperationsvorhaben, die eine konkrete Organisationsform für inter-institutionelle Zusammenarbeit finden und festigen konnten. Sie hatten insoweit eine Pionierrolle (WiBIG 2004 d).

Es hat sich gezeigt, dass Interventionsprojekte eine geeignete Organisationsform sind, um das Thema häusliche Gewalt auf verschiedenen Ebenen zu bearbeiten. Die Verbesserungen im Problemfeld häuslicher Gewalt, die durch die Interventionsprojekte angestoßen und erreicht werden konnten, liegen

- auf der Ebene von Gesetzen und Erlassen. Diese fest verankerten Ergebnisse (Polizeigesetze, Gewaltschutzgesetz, etc) bestimmen sowohl das Handeln wichtiger relevanter Institutionen als auch den öffentlichen Diskurs über häusliche Gewalt nachhaltig, ferner

- auf der Ebene von Organisationsstrukturen. Große staatliche Institutionen wie die Polizei oder Amts- und Staatsanwaltschaften haben Sonderzuständigkeiten für häusliche Gewalt in ihrer Struktur verankert. Das Thema konnte in Aus- und Fortbildungen verankert werden. Das Unterstützungssystem konnte um zugehende Angebote ergänzt werden, und es wurden Wege gefunden, Täter zur Veränderung ihres Gewaltverhaltens aufzufordern.

- Außerdem finden sie auf der Ebene des persönlichen Engagements, Denkens und Handelns relevanter Akteure statt.

Interventionsprojekte haben sich darüber hinaus als geeignet erwiesen, gewachsene konträre Sichtweisen zu verschränken. Dies zeigt sich exemplarisch in den Feldern Frauenschutz und Kinderschutz. Vertreterinnen und Vertreter von Jugendämtern, Familiengerichten und Kinderschutzeinrichtungen als Kooperationspartner zu gewinnen, gehörte und gehört zu den schwersten Aufgaben der Kooperationsbündnisse. Das Arbeitsfeld der Unterstützung von Frauen bei häuslicher Gewalt stellt die Frauen als Gewaltopfer in den Mittelpunkt, definiert Gewalt auf dem Hintergrund der Analyse des Geschlechterverhältnisses als Männergewalt und sieht Kinder und Jugendliche als Mitbetroffene in diesem Kontext. Das Arbeitsfeld des Kinderschutzes und der Jugendhilfe stellt Kinder und Jugendliche als Gewaltopfer in den Mittelpunkt, definiert Gewalt auf dem Hintergrund des Generationenverhältnisses als Gewalt durch Erwachsene und sieht Frauen als potenzielle Täterinnen in diesem Kontext. Es mussten historisch bedingte Gräben überwunden werden und die Arbeit mit unterschiedlichen Opfergruppen miteinander verknüpft werden. Dies wurde vielerorts begonnen. Damit wurde ein Raum dafür eröffnet, die Betroffenheit der Kinder von Gewalt gegen die Mutter (und ggf. auch durch die Mutter) erstmals an einem Ort zum Thema zu machen. Dem Ziel der Interventionsprojekte, mehr Kooperation zwischen den Beratungs- und Schutzeinrichtungen für Frauen einerseits und denen für Kinder andererseits zu etablieren, wurde näher gekommen.

Die Arbeit der Interventionsprojekte hat Ausstrahlungswirkung in andere Arbeitsfelder. Die Idee verbindlicher inter-institutioneller Kooperation bei häuslicher Gewalt könnte beispielsweise in den Gesundheitsbereich hineingetragen werden. Auch wenn noch nicht absehbar ist, ob sich das Arbeitsmodell »Interventionsprojekt « dort als die geeignete Form für Kooperation erweisen wird, so ist deutlich geworden, dass vielfältig und an vielen Orten versucht wurde, in der gesundheitlichen Versorgung eine breitenwirksame Anlaufstelle für eine eigene »Interventionskette« zu schaffen, und dass Initiative und Verantwortung hierfür nicht einer gesundheitlichen Institution überlassen wurde; Vertrauen wurde vielmehr in den Weg der inter-institutionellen Kooperation gesetzt. Dies wäre einer der 
denkbaren Ausstrahlungseffekte der hier beschriebenen Projekte.

Die Interventionsprojekte haben insgesamt mit ihrer Organisationsstruktur und demokratischen Arbeitsweise einen gangbaren Weg für die Bearbeitung von Querschnittsthemen gebahnt. Sie können deshalb Modell für gesellschaftliche Veränderung auch in anderen Problemfeldern sein, für die sich ein interdisziplinärer und interinstitutioneller Zugang anbietet.

Interventionsprojekte haben den Weg zur Entwicklung von Standards guter Praxis bei häuslicher Gewalt geebnet. Durch das koordinierte inter-institutionelle Vorgehen und die Entwicklung modellhafter guter Praxis konnte eine Basis der Verständigung über häusliche Gewalt erreicht werden, die die Entwicklung und Akzeptanz von Standards guter Praxis ermöglicht.

\subsection{Beratung nach einem Polizeieinsatz wegen häuslicher Gewalt}

Durch die veränderte polizeiliche Praxis ist großer Beratungsbedarf entstanden, der neue Anforderungen an Beratung stellte.

Die staatliche Intervention bleibt für die von häuslicher Gewalt Betroffenen und ihre Kinder nicht ohne Folgen und entspricht nicht immer den meist unklaren Erwartungen oder Wünschen, die Betroffene an Intervention haben. Sie kann sogar Krisen verschärfen oder auslösen, wenn Entscheidungen getroffen werden müssen oder Handlungsbedarf entsteht. Vielerorts setzte sich deshalb die Einschätzung durch, dass nicht gewartet werden kann, bis Gewaltopfer von sich aus Beratungsstellen oder Frauenhäuser aufsuchen, sondern dass ihnen offensiver Information und Beratung angeboten werden müsse. Beispiele aus dem Ausland ermutigten diejenigen, die in Unterstützungseinrichtungen oder bei der Polizei dieses Ziel verfolgten. Die KommStruktur der Unterstützungslandschaft bei häuslicher Gewalt erfuhr daher Ergänzungen.

Im Rahmen der wissenschaftlichen Begleitung von Interventionsprojekten konnte die Entwicklung innovativer Beratungsangebote bei häuslicher Gewalt mitverfolgt werden. Die hier vorgestellten Ergebnisse resultieren aus der Evaluation zweier neuartiger, zugehender Formen der Unterstützung (WiBIG 2004 a). In MecklenburgVorpommern wurden Interventionsstellen mit pro-aktiver Beratung eingerichtet. Im Anschluss an polizeiliche Intervention bei häuslicher Gewalt kontaktieren sie nach automatischer Datenweitergabe unmittelbar die Betroffenen und bieten Beratung und Krisenintervention an. Sie wurden - angelehnt an das österreichische Vorbild - als Bindeglied zwischen polizeilicher Intervention und zivilrechtlichen Schutzmöglichkeiten eingerichtet. In Berlin hat die BIG-Hotline eine Mobile Intervention geschaffen, die in Kri- sensituationen aufsuchende Beratung und Unterstützung für Frauen anbietet. Mobile Intervention wird täglich von 9.00 bis $24.00 \mathrm{Uhr}$ bereit gehalten. Auf Wunsch erfolgt eine Beratung der Frauen in ihrer Wohnung oder an einem anderen Treffpunkt; sie wurde häufig im Kontext polizeilicher Intervention angefragt.

Beide Angebote, die Interventionsstellen und die Mobile Intervention, fasst die Begleitforschung als zugehende Angebote zusammen, weil sie Opfer häuslicher Gewalt von den Mühen des Weges (Mobile Intervention) oder des Schrittes der Kontaktaufnahme (Interventionsstellen) entlasten. ${ }^{15}$

- Die zugehenden Unterstützungsangebote sind geeignet, den Bedarf zu decken, der durch veränderte polizeiliche Praxis und zivilrechtliche Möglichkeiten entsteht.

Befürchtungen, dass betroffene Frauen den pro-aktiven Ansatz ablehnen oder sich dieser destruktiv auswirken könnte, weil die Betroffenen sich entmündigt oder erneut zum Opfer gemacht fühlen, haben sich nicht bestätigt. Im Gegenteil erwies sich die pro-aktive Beratung als Beitrag zur Bestärkung der Betroffenen im Sinne einer Erweiterung der Handlungs- und Entscheidungsspielräume, einer Erhöhung der Selbstmächtigkeit und des Rückgewinns von Kontrolle über das eigene Leben.

Nur 35 der 809 Klientinnen und Klienten der Interventionsstellen, zu denen die Kontaktaufnahme gelang (5\%), lehnten eine Beratung ausdrücklich ab. Der Zeitpunkt der Kontaktaufnahme scheint von Bedeutung zu sein. Je später die Kontaktaufnahme gelang, desto häufiger wurde die Beratung abgelehnt: In 23 von 35 Fällen waren bereits drei bis sieben Tage seit dem Polizeieinsatz verstrichen.

Pro-aktiver Beratung gelang es, auch diejenigen von häuslicher Gewalt Betroffenen mit Information und Unterstützung zu versorgen, die von sich aus keinen Unterstützungsbedarf an Beratungsstellen herantragen würden. Sei es,

- weil sie sich subjektiv nicht als unterstützungsbedürftig sehen, obwohl sie Gewalt erleiden

- und ihnen Informationen über ihre rechtlichen Möglichkeiten fehlen,

- weil sie glauben, dass ihr Fall "nicht schlimm genug« ist, um Unterstützung zu »verdienen ",

- weil sie verängstigt oder resigniert sind, nicht mehr aktiv Hilfe suchen bzw. nicht mehr auf Hilfe hoffen.
- Die zugehenden Unterstützungsangebote erreichen neue Zielgruppen.

Den neuen Angeboten gelang die Kontaktaufnahme auch zu von häuslicher Gewalt Betroffenen, die keine Information über Möglichkeiten der Intervention und des Schutzes haben und die ihre Rechte ebenso wenig kennen wie das örtliche Beratungsangebot. Die Auswertung der Beratungsdokumentationen in den Interventionsstellen ergab beispielsweise, dass mehr als ein Drittel der Betroffenen bezüglich der häuslichen Gewalt zuvor keinen anderen Kontakt zu irgendwelchen Beratungs- oder Unterstützungseinrichtungen hatte.

Zugehende Beratung hat sich auch sehr für die Zielgruppe von Migrantinnen - insbesondere denjenigen mit geringen Deutschkenntnissen bewährt. Die erforderliche Sprachmittlung kann sehr viel einfacher organisiert werden, wenn die Nachfrage nach Beratung nicht unerwartet kommt, sondern der Zeitpunkt von der Beraterin bestimmt werden kann. Wurde bereits auf dem Fax der Polizei angegeben, dass Sprachmittlung erforderlich ist, kann eine Dolmetscherin die Beraterin im Einsatz begleiten oder telefonisch zugeschaltet werden. Auch dem Beratungsbedarf derjenigen Migrantinnen, die in sozialer Isolation leben und die Strukturen des deutschen Hilfesystems nicht kennen, kann so gut entsprochen werden.

- Zugehende Angebote brauchen eine Einbettung in ein Unterstützungssystem, andere Einrichtungen wie Frauenhäuser werden dadurch nicht überflüssig.

Die neuen zugehenden Beratungsangebote ergänzen bestehende Einrichtungen und können sie nicht ersetzen. Im Gegenteil: Sie sind auf sie angewiesen. Stationäre Angebote wie Frauenhäuser wurden nicht überflüssig, stattdessen ebneten der pro-aktive Ansatz und die aufsuchende Beratung vielen Frauen den Weg dorthin, die ihn aus eigener Kraft nicht gefunden hätten.

Überall dort, wo pro-aktiv und aufsuchend gearbeitet wird, werden auch Betroffene erreicht, für die die polizeilichen und/oder juristischen Schutzmaßnahmen nicht ausreichen. Sie benötigen die Möglichkeit, in einer Schutzeinrichtung unterzukommen, z.B. weil sie so geängstigt sind, dass sie kurzzeitig an einen sicheren Ort gehen, von dem aus sie in ihre Wohnung zurückkehren, sobald sie die Krise durchstanden bzw. Sicherheit organisiert haben, oder weil sie anhaltend bedroht sind, und aus diesem Grund an einen sicheren Ort flüchten müssen, von dem aus sie längerfristige Schutzmaßnahmen beantragen und/oder sich eine neue Wohnung suchen.

- Die zugehenden Unterstützungsangebote decken strukturelle Barrieren des Hilfesystems auf und senken die Schwellen. Sie tragen zum 
Funktionieren der Interventionskette bei und helfen, deren Schwachstellen zu erkennen und nachzubessern.

Aufsuchende Krisenintervention sowie Begleitung sehen Betroffene in ihrem Lebensumfeld und gehen die Wege der Hilfesuche mit ihnen gemeinsam. Auf diese Weise kann ein Einblick aus der Perspektive der Betroffenen gewonnen werden, welche Veränderungen hilfreich sein können und nach welchen subjektiven Kriterien die Stationen der Interventionskette genutzt bzw. nicht genutzt werden. Strukturelle Barrieren des Hilfesystems wie fehlende Sprachmittlung, komplizierte bürokratische Vorgänge oder unklare Zuständigkeiten werden durch die Begleitung aufgedeckt.

Die neuen Angebote senken die Schwelle ins Hilfesystem und verbessern die Chancen der Betroffenen, die Form der Beratung oder des Schutzes zu wählen, die ihren Bedürfnissen, ihrer Gefährdung und ihrer Lebenssituation entspricht.

\section{- Zugehende Beratungsangebote entlasten die} Polizei.

Die Polizei sieht in den akuten Einsatzsituationen oft einen großen Unterstützungsbedarf der Betroffenen und ihrer Kinder. Diesen zu decken, liegt weder in ihrer Kompetenz, noch ist es ihr Auftrag. Der Bedarf seitens der Polizei an jederzeit abrufbarer Krisenintervention und Beratung für die Betroffenen ist groß. Dort, wo es dieses Angebot nicht gibt, wird von der Polizei oft versucht, es auf Umwegen, z.B. in bilateralen Absprachen mit einzelnen Stellen, zu erreichen.

\subsection{Staatliche Intervention bei häuslicher Gewalt durch Polizei und Staatsanwalt- schaft}

Lange Zeit war die Bearbeitung der Fälle häuslicher Gewalt durch die Polizei und Amts- bzw. Staatsanwaltschaften geprägt von der Haltung, dass Gewalt, die in privaten Beziehungen stattfindet, auch dort geregelt werden sollte. Häusliche Gewalt wurde als Familienstreitigkeit definiert. Die Polizei reagierte auf strafrechtlich relevante Delikte wie Körperverletzung, Drohungen oder Nötigung überwiegend mit dem Versuch einer Streitschlichtung. Sie bearbeitete die Fälle, wenn überhaupt, im so genannten vereinfachten Ermittlungsverfahren, bei dem Geschädigte und Beschuldigte nicht persönlich vernommen, sondern lediglich aufgefordert werden, sich schriftlich zu äußern.

Eine Arbeitsroutine in den Amts- und Staatsanwaltschaften war die Verweisung der Verfahren auf den Privatklageweg. Der Staat bescheinigt damit den Geschädigten ein mangelndes öffentliches Interesse an der Strafverfolgung und überlässt die Strafverfolgung ihrer privaten
Initiative. Es herrschte ein deutliches Vollzugsdefizit bestehender Gesetze.

Zur Einschätzung der »neuen « Praxis hat die wissenschaftliche Begleitung vielfältige Erhebungen durchgeführt, beispielsweise schriftliche Befragung von Polizeibeamten und -beamtinnen zu Einsätzen häuslicher Gewalt, schriftliche Befragung von Frauen zu Polizeieinsätzen, Auswertung von insgesamt 959 Strafakten in zwei Sonderdezernaten der Amtsanwaltschaften Berlin und Flensburg (WiBIG 2004 b).

Die Polizei nimmt häusliche Gewalt als Straftat wahr und ist zunehmend bereit, auf der Grundlage der Leitlinien täterorientiert zu intervenieren. Anzeichen dafür, dass häusliche $\mathrm{Ge}$ walt von polizeilicher Seite nicht länger als Privatangelegenheit betrachtet wird, sind $u$. a. ein veränderter polizeiinterner Sprachgebrauch, Präferenzen bei der Wahl polizeilicher Interventionsmittel sowie die Akzeptanz der neuen Begrifflichkeiten und Eingriffsmaßnahmen. Die Maßnahme der Wegweisung des Täters/der Täterin bei häuslicher Gewalt aus der Wohnung ist Bestandteil neuer polizeilicher Praxis geworden.

- Die polizeiliche Praxis zeigt Verbesserungsbedarf im Bereich der Beweissicherung.

Dies zeigte sich daran, dass Fälle häuslicher Gewalt weiterhin im vereinfachten Ermittlungsverfahren bearbeitet wurden: D.h., in $11 \%$ bis $65 \%$ der Verfahren haben entweder die Beschuldigten oder die Geschädigten keine Ladung zu einer persönlichen Vernehmung bekommen. Dieses Verfahren wird von Expertinnen und Expertinnen der Polizei und Amtsanwaltschaft als nicht angemessen bewertet. Die Aufforderung an Beschuldigte und Geschädigte, Umstände und Verlauf des angezeigten Geschehens in Schriftform zu verfassen, stellt häufig eine Hemmschwelle dar, zu reagieren. Unterschiedliche Sprachkompetenzen und Bildungsniveaus erhöhen diese Barriere.

Die Polizei hat zum Teil nach einem Einsatz keine weiteren Schritte der Beweiserhebung unternommen. Beschuldigte und Geschädigte erhielten in diesen Fällen weder eine Aufforderung zur schriftlichen Stellungnahme, noch wurden sie am Tatort vernommen oder wurden zur späteren Vernehmung auf eine Polizeidienststelle geladen. Ein Einstellungsbescheid von der Amtsanwaltschaft war die nächste behördliche Reaktion nach der Anzeigenerstattung.

Zum Zeitpunkt der Erhebungen hat die Polizei kaum Fotos von Verletzungen oder dem Tatort gefertigt und nur wenige Spontanäußerungen notiert. Je nach Untersuchung fotografierten in $1 \%$ bis $3 \%$ der Verfahren die Beamten/ Beamtinnen Verletzungen von Geschädigten. Bei einer Quote von insgesamt über 80\% Kör- perverletzungsdelikten besteht hier ein hohes Verbesserungspotenzial.

Auch Beweismittel wie Spontanäußerungen, die in Kombination mit Aussagen von Beamten und Beamtinnen u. U. eine Aussage der geschädigten Person vor Gericht entbehrlich machen könnte, hatte die Polizei nur sehr selten notiert.

Bei der Umsetzung koordinierter Intervention in Fällen häuslicher Gewalt konnte die Ebene der Strafverfolgung bisher noch nicht in gleichem Maße eingebunden werden wie die Ebene der Gefahrenabwehr durch die Polizei.

An der Schnittstelle zur Strafverfolgung endet in vielen Fällen immer noch die staatliche Intervention, während Interventionsprojekte eine lückenlose Interventionskette vom polizeilichen Einsatz bis zu zivilrechtlichen Schutzmöglichkeiten wünschen. Aber dies gelang bislang nicht ausreichend, schon gar nicht gelang es die Strafverfolgung an diesem Prozess zu beteiligen und in ein Gesamtkonzept zur (staatlichen) Intervention bei häuslicher Gewalt einzubinden.

- Eine Sanktionierung häuslicher Gewalt durch die Amtsanwaltschaft findet selten statt. Das Ziel eines entscheidenden Richtungswechsels hin zu konsequenter Strafverfolgung kann bisher nicht umgesetzt werden.

Die Untersuchung von Ermittlungsverfahren aus zwei verschiedenen Amtsanwaltschaften hat gezeigt, dass die überwiegende Anzahl der Verfahren eingestellt wurde. In 95,8\% (347) bzw. $81,7 \%$ (488) der Verfahren haben die Dezernentinnen/Dezernenten keine öffentliche Klage gegen die Beschuldigten erhoben. In beiden Untersuchungen wich die Quote von der der behördeninternen Statistik ab, denn beide Behörden wiesen einen geringeren Anteil an Einstellungen aus. Hiernach beliefen sich die Zahlen auf $69,8 \%$ bzw. 89,8\%. Festzuhalten bleibt aber, dass selbst, wenn man die niedrigste offizielle Zahl der Einstellungen zugrunde legt, die Amtsanwaltschaften mindestens zwei Drittel der Verfahren häuslicher Gewalt eingestellt haben.

Die Zahlen umfassen alle Arten von Einstellungen. Die am häufigsten gewählte Form der Einstellung des Verfahrens war die nach $\S 170$ Abs. 2 Strafprozessordnung.

- Die Umsetzung der Forderung, in der Regel das besondere öffentliche Interesse zu bejahen und Verfahren häuslicher Gewalt nicht auf den Privatklageweg zu verweisen, schafft einen adäquaten prozessualen Rahmen für diese Verfahren, löst aber allein das Problem der hohen Einstellungsquoten nicht.

Ein deutlicher Unterschied der beiden Untersuchungen zeigte sich in der Frage, warum die 
Dezernentinnen/Dezernenten Täter nicht anklagten. Die eine Amtsanwaltschaft handelte im Rahmen ihrer Dienstanweisung. Es wurden dort in der Regel das besondere öffentliche Interesse an der Strafverfolgung bejaht und kaum Fälle auf den Privatklageweg verwiesen. Diese Aspekte spielten für die Einstellung der Verfahren keine Rolle mehr. Hier lautete der am häufigsten genannte Grund für eine Einstellung nach § 170 Abs. 2 StPO die mangelnde Nachweisbarkeit des Tatvorwurfs (83\%, 342 der 408 Einstellungen nach $\S 170$ Abs. 2 StPO).

Die Dezernentinnen/Dezernenten der anderen Amtsanwaltschaft haben ihre Einstellung überwiegend damit begründet, dass ein besonderes öffentliches Interesse nicht vorliege oder in einem Drittel der Fälle die Verfahren auf den Privatklageweg verwiesen.

Trotzdem ist in beiden Untersuchungen der Anteil der Verfahrenseinstellungen ähnlich. Die veränderte Verfahrensweise der einen Amtsanwaltschaft konnte entgegen den Erwartungen das Problem hoher Einstellungsquoten nicht lösen.

- Der Zusammenhang zwischen der Entscheidung der Geschädigten zur Mitwirkung an einem Strafverfahren und dem Verfahrensausgang ist signifikant. Bei der Bearbeitung der Fälle folgt der Lauf des Verfahrens in der Regel dem vermeintlichen Willen der Geschädigten.

In vielen Fällen lehnten es Dezernentinnen/Dezernenten ab, gegen den Willen der geschädigten Person ein Strafverfahren zu führen; sie interpretierten das Ausbleiben einer Mitwirkung an der Strafverfolgung als Willensäußerung der Geschädigten. Aspekte wie die Sicherheit der Geschädigten, Wahrung ihrer Entscheidung, aber auch mangelnde Strafwürdigkeit spielten hierbei eine Rolle. Zudem werteten die Dezernentinnen/Dezernenten das Beweismittel der Zeugenaussage der Geschädigten in den meisten Fällen als unverzichtbar für die Erhebung der öffentlichen Klage.

Die Ergebnisse weisen auf eine hohe Ambivalenz der Geschädigten in Bezug auf ein Strafverfahren hin und lässt vermuten, dass sie bei entsprechenden Anstrengungen häufiger als bisher zur Mitwirkung im Verfahren gewonnen werden könnten.

\subsection{Täterarbeit im Kontext von Interventionsprojekten}

Mit dem Entstehen von Kooperationsbündnissen gegen häusliche Gewalt wurde verstärkt gefordert, Täter häuslicher Gewalt für ihre Gewalthandlungen in die Verantwortung zu nehmen. Täterprogramme sind in Deutschland ein relativ neues und innovatives Arbeitsfeld mit einer sehr dynamischen Entwicklung. Die Einbin- dung von Täterarbeit in Kooperationsbündnisse gegen häusliche Gewalt nimmt seit 2002 stark zu. Einige wenige Einrichtungen können mittlerweile auf mehrere Jahre Erfahrung mit dem Angebot von sozialen Trainingskursen im Kontext von Interventionsprojekten zurück blicken. Viele stehen aber damit erst am Anfang.

Zielgruppe der begleiteten Täterprogramme waren ausschließlich Männer. Deren konzeptionelle Ausgestaltung variierte in den einzelnen Programmen. Verbindliche bundesdeutsche Standards für die Täterarbeit sind in der Entwicklung. Es handelt sich bei Täterarbeit um Maßnahmen, die gezielt und strukturiert Gewalthandlungen von Männern gegenüber ihren (Ex)Partnerinnen bearbeiten. Die hierbei verwendete Methodik ist vielseitig und noch am ehesten als kognitiv-verhaltenstherapeutisch orientiert zu bezeichnen. Die von WiBIG evaluierten Kurse umfassten derzeit zwischen 12 und 26 Sitzungen, die wöchentlich in Gruppenform stattfanden.

Wenn auch nicht einheitlich in Deutschland, so gelangte speziell im Kontext von Interventionsprojekten die justizielle Weisung doch auf verschiedenen rechtlichen Grundlagen zur Anwendung und gewann langsam an Bedeutung. Größte praktische Relevanz haben zurzeit Auflagen auf der Ebene von Amts- und Staatsanwaltschaften $^{16}$ (WiBIG 2004 c).

Die Teilnehmer, die über eine justizielle Weisung in die Programme gelangt sind, waren in der Regel Beschuldigte eines Ermittlungsverfahrens wegen Körperverletzung. Der am häufigsten genannte Tatvorwurf war der der einfachen Körperverletzung, ein Drittel der Teilnehmer wurden der gefährlichen Körperverletzung beschuldigt.

- Knapp zwei Drittel der Männer, die ein Programm begannen, schlossen es ab.

Von 322 Tätern, die mit den Einrichtungen Kontakt aufgenommen haben, lagen sozialstatistische Daten vor. Von ihnen haben 213 ein Täterprogramm begonnen, davon haben 137 (64\%) das Programm abgeschlossen. 76 Teilnehmer schlossen die Kurse nicht ab, von ihnen brachen 56 das Programm von sich aus ab, und 20 wurden von den Einrichtungen ausgeschlossen. 77\% der Abbrüche erfolgten in der ersten Kurshälfte.

Von allen dokumentierten Tätern häuslicher Gewalt (322) verweigerten 29\% (94) die Teilnahme und weitere $5 \%$ (15) wurden von den Einrichtungen nicht zum Programm zugelassen.

- Die Teilnehmer der sozialen Trainingskurse zeichneten sich durch ein verhältnismäßig niedriges Niveau der Schulbildung aus.

Gut die Hälfte der dokumentierten Täter (52\%) verfügte über einen Hauptschulabschluss.
Weitere $12 \%$ besaßen keinen Schulabschluss. Nimmt man für die Bundesrepublik das Bildungsniveau als Indiz für soziale Schicht, so lassen die Daten folgendes Muster erkennen: Der Zugang zu einem Täterprogramm, das im Kontext polizeilicher Intervention und Justiz arbeitet, wurde vorrangig Männern aus den unteren sozialen Schichten vermittelt. Sie werden vermutlich überproportional häufiger für häusliche Gewalt erfasst und sanktioniert. Hingegen verblieben Männer mit einem Mittelschichtshintergrund deutlich häufiger in den Kursen und schlossen sie ab. Hier war Potenzial für Verbesserungen erkennbar: Einerseits könnten Polizei und Justiz aufmerksamer darauf achten, dass häusliche Gewalt in der Mittelschicht ebenso wahrgenommen und sanktioniert wird. Die Einrichtungen, die Täterarbeit anbieten, könnten andererseits ihre Kurse konzeptionell weiterentwickeln, um erfolgreicher mit Männern aus eher bildungsfernen Milieus zu arbeiten.

- Sozialstatistische Merkmale wirkten sich förderlich auf den Abschluss eines Täterprogramms aus.

Der Zusammenhang zwischen folgenden Faktoren und einem Abschluss des Programms hat sich als statistisch signifikant erwiesen.

- Niveau des Schulabschlusses: Teilnehmer mit einem höheren Schulabschluss haben die Programme häufiger abgeschlossen. Je höher der Schulabschluss, desto größer war die Wahrscheinlichkeit, das Programm abzuschließen.

- Einkommen: Männer, die das Programm abgeschlossen haben, bestritten häufiger ihren Lebensunterhalt auf der Grundlage einer eigenen Arbeitsleistung als diejenigen, die das Programm abgebrochen hatten.

- Täter, die aufgrund einer justiziellen Weisung oder Auflage an einem Täterprogramm teilnahmen, schlossen dieses signifikant häufiger $\mathrm{ab}$ als Teilnehmer ohne einen justiziellen Hintergrund.

Eine justizielle Weisung kann offenbar die Motivation steigern, die Anforderungen einer Täterprogrammteilnahme durchzuhalten. Männer, die gegenüber ihren Partnerinnen gewalttätig geworden sind, melden sich nur selten aus eigener Motivation in Einrichtungen, die verhaltensändernde Maßnahmen für diese Zielgruppe anbieten. Oftmals ist gerade deshalb ein äußerer Druck nötig, ein Täterprogramm aufzunehmen.

WiBIG konnte keine Wirksamkeitsforschung durchführen, aber aus der Sicht der Gruppenleitungen zeigten die meisten Teilnehmer positive Veränderungen in ihrer Einstellung zu ihrer (Ex)Partnerin oder gegenüber Frauen im Allgemeinen. 


\section{Themen für die Zukunft}

Für die Arbeit der Interventionsprojekte bedeutet das für die Zukunft zum einen, die erzielten Ergebnisse zu sichern und zum anderen in die Phase der Weiterentwicklung und Feinabstimmung neuer Maßnahmen und Kooperationswege einzusteigen.

Im Bereich der Unterstützungsangebote für von häuslicher Gewalt Betroffene geht es zukünftig darum, die Versorgung mit proaktiver Erstberatung auszubauen sowie das Angebot an Schutz- und Beratungseinrichtungen sicherzustellen. Darüber hinaus sollten Schwellen im Unterstützungssystem gesenkt werden, um beratungsferne Zielgruppen wie Migrantinnen mit geringen Deutschkenntnissen, mitbetroffene Kinder und Jugendliche sowie bildungsferne Gruppen zu erreichen.

Am Ende der Evaluationslaufzeit im Bereich der Amtsanwaltschaften zeigte sich auf mehreren Ebenen Klärungsbedarf:

- Wie wollen die Strafverfolgungsbehörden mit der oft ausbleibenden Kooperation durch die Geschädigten in ihrer Funktion als Zeuginnen und Zeugen umgehen?

- Wie kann Unterstützung für die Zeuginnen und Zeugen zu einem frühen Zeitpunkt des Ermittlungsverfahrens eingebunden werden?

- Wie kann organisiert werden, dass Amtsund Staatsanwaltschaften einen größeren Fokus auf den Kontakt zu den Geschädigten, den potenziellen Zeuginnen/Zeugen, legen?

Im Bereich der Umsetzung der mittlerweile nicht mehr neuen polizeilichen Maßnahme der Wegweisung gibt es Hinweise darauf, dass eine nicht zu unterschätzende Anzahl von Tätern die Anordnung, die Wohnung für eine Zeit nicht mehr zu betreten, unterläuft. Hier herrscht zum einen Unklarheit, inwiefern der Wille der Geschädigten bei einer konsequenten Weiterführung der Maßnahme zu berücksichtigen ist. Zum anderen scheint es ein Handlungsvakuum für die Fälle der »unbeeindruckbaren« Hochrisikotätern zu geben.

Für diese Fälle besteht Bedarf an einer steigerungsfähigen, differenzierter polizeilichen Interventionsstrategie (WiBIG b, S. 241)

Amts- und Staatsanwaltschaften sollten verstärkt in Täterprogramme weisen. Hier bedarf es einer Verbesserung der Kooperation. Ein Schwachpunkt ist bislang die Einbettung in eine konsequente Strafverfolgung. Sowohl bei Nichtteilnahme als auch bei Abbruch der Maßnahme erfolgt eine Sanktionierung noch nicht regelmäßig.

\section{Literatur:}

Dearing, Alwin/Haller, Birgitt 2000: Das österreichische Gewaltschutzgesetz, Juristische Schriftenreihe Band 163, Wien

Jungnitz, Ludger/Lenz, Hans-Joachim u.a. 2004: Gewalt gegen Männer, BMFSFJ (Hg.)

Kriminologische Forschungsinstitut Niedersachsen, Rebecca Löbmann, Karin Herbers 2004: Mit BISS gegen häusliche Gewalt - Evaluation des Modellprojektes "Beratungs- und Interventionsstellen (BISS) für Opfer häuslicher Gewalt in Niedersachsen«, Niedersächsisches Ministerium für Soziales, Frauen, Familie und Gesundheit (Hg.)

Müller, Ursula/Schröttle, Monika 2004: Lebenssituation, Sicherheit und Gesundheit von Frauen in Deutschland. Eine repräsentative Untersuchung zu Gewalt gegen Frauen in Deutschland, BMFSFJ (Hg.)

WiBIG (2004 a): Neue Unterstützungspraxis bei häuslicher Gewalt, BMFSFJ (Hg.) www.bmfsfj.de Stichwort? Forschungsnetz? Forschungsberichte

WiBIG (2004 b):Staatliche Intervention bei häuslicher Gewalt, BMFSFJ (Hg.) www.bmfsfj.de Stichwort? Forschungsnetz? Forschungsberichte

WiBIG (2004 c): Täterarbeit im Kontext von Interventionsprojekten gegen häusliche Gewalt, BMFSFJ (Hg.) www.bmfsfj.de Stichwort ? Forschungsnetz ? Forschungsberichte

WiBIG (2004 d): Von regionalen Innovationen zu Maßstäben guter Praxis - Die Arbeit von Interventionsprojekten gegen häusliche Gewalt, BMFSFJ (Hg.) www.bmfsfj.de Stichwort ? Forschungsnetz ? Forschungsberichte

Die Verfasserinnen sind tätig beim Sozialwissenschaftlichen Frauen Forschungs Institut der Kontaktstelle praxisorientierte Forschung e.V. SoFFI.K.Berlin - Büro Berlin, Kottbusser Damm 79, 10967 Berlin, Tel: 030/691 4832

\section{Fußnoten:}

1 Das österreichische Gewaltschutzgesetz trat am 1.05.1997 in Kraft und umfasste Änderungen im Sicherheitspolizeigesetz und der so genannten Exekutionsordnung.

2 Gesetz zur Verbesserung des zivilrechtlichen Schutzes bei Gewalttaten und Nachstellungen sowie zur Erleichterung der Überlassung der Ehe Wohnung bei Trennung, in Kraft getreten am 1.1.2002.

3 Da sich die überwiegende Anzahl der Ländergesetzgebungen im Bereich des Polizeirechts stark ähnelt, sehen die meisten Gesetze für den Bereich der häuslichen Gewalt mittlerweile Standardmaßnahmen »Wegweisung / Betretungsverbot « vor, die es der Polizei erlauben, Täter häuslicher Gewalt je nach Bundesland in einem ersten Schritt zwischen 10 und 14 Tagen aus der Wohnung zu verweisen.

4 Die wissenschaftliche Begleitung wurde von der Forschungsgruppe WiBIG an der Universität Osnabrück unter der Leitung von Carol HagemannWhite und Barbara Kavemann durchgeführt.

5 Berlin: Berliner Interventionszentrale bei häuslicher Gewalt - BIG mit der BIG-Hotline und der Mobilen Intervention sowie zwei in Berlin angebotene Täterprogramme, Bremen: Neue Wege e.V.,

Freiburg: Freiburger Interventionsprojekt gegen häusliche Gewalt - FRIG, Gladbeck: Gladbecker Initiative gegen Gewalt in Familien - GIP, Hannover: Hannoversches Interventionsprojekt gegen MännerGewalt in der Familie - HAIP, MecklenburgVorpommern: Interventionsprojekt CORA - Contra Gewalt gegen Frauen und Mädchen in Mecklenburg, Passau: Gewalt im sozialen Nahraum, Rheinland-Pfalz: Rheinland-Pfälzisches Interventionspro- jekt gegen Gewalt in engen sozialen Beziehungen RIGG, Schleswig-Holstein: Kooperations- und Interventionskonzept des Landes Schleswig-Holstein gegen häusliche Gewalt an Frauen - KIK-SchleswigHolstein mit mittlerweile 12 regionalen KIKs, Stuttgart: STOP - Stuttgarter Ordnungspartnerschaft gegen häusliche Gewalt.

6 als Teil des Interventionsprojektes CORA.

7 Erlass der Frauen- und Gleichstellungsbeauftragten vom 05.02.2002, Amtsblatt für Mecklenburg-Vorpommern 2002 Nr.11, S. 241.

8 Die Dokumentation des internationalen Kongresses, auf dem die Ergebnisse präsentiert wurden, ist auf der Internetseite des BMFSFJ abzurufen.

9 Sie wurde an der Universität Bielefeld unter der Leitung von Ursula Müller und Monika Schröttle durchgeführt und von einem international besetzen Beirat unterstützt.

10 Vergleichbare Studien liegen vor ais den Niederlanden, der Schweiz, England und Wales, Dänemark, Irland, Finnland und Schweden (Literaturhinweise siehe BMFSFJ 2004 e).

11 Sie wurde von einer interdisziplinären Forschergruppe durchgeführt unter der Beteiligung von Dissens e.V. Berlin, SoKo Bielefeld und GeFoWe Eckenhaid und von einem interdisziplinären wissenschaftlichen Beirat unterstützt.

12 Vorgelegt wurden darüber hinaus im April 2005 die Ergebnisse der vom Niedersächsischen Sozialministerium in Auftrag gegebenen und vom Kriminologischen Forschungsinstitut Niedersachsen (Karin Herbers und Rebecca Löbmann) durchgeführten Evaluation der Interventionsstellen in Niedersachsen sowie die Ergebnisse der an der TU Berlin durchgeführten wissenschaftlichen Begleitung des SIGNAL- Interventionsprogramms »Häusliche Gewalt gegen Frauen: gesundheitliche Versorgung (Hellbernd/Brzank u.a. 2003). Erwartet werden in diesem Jahr die Ergebnisse der vom Bundesministerium für Justiz in Auftrag gegebenen und von der Universität Bamberg (Marina Rupp) durchgeführten Evaluation des Gewaltschutzgesetzes.

13 Der Austausch über alle drei Studien wird in internationalen Forschungsnetzwerken geführt wie European Network on Gender, Conflict and Violence und CAHRV - Coordination Action on Human Rights Violations.

14 Die ausführlichen Ergebnisse wurden im Abschlussbericht WiBIG 2004, Band a-d veröffentlicht. Er ist im Internet unter www.bmfsfj.de (Stichwort $\ddagger$ Forschungsnetz $\ddagger$ Forschungsberichte) abrufbar.

15 Die Erkenntnisse basieren unter anderem auf der Auswertung von 1194 Beratungsverläufen in den fünf Interventionsstellen, einer schriftliche Klientinnenbefragung (87 Fragebögen) sowie der Auswertung von 335 Anfragen und 240 durchgeführten Einsätzen der Mobilen Intervention.

16 In die Auswertung gingen insgesamt 1.201 Erhebungsbögen (Klienten- und Gruppenverlaufsbögen) ein sowie Interviews mit Gruppenleitungen, Teilnehmern und Partnerinnen ein. 